\title{
Patient with alopecia areata
}

\section{Derkowska Ilona, Korzenlowska Katarayna, Brandt Agnieszka, Buraczewska Marta, Mysiliwiec Malgorzata}

Medioal University of Cdańsk, Poland, Glinios of Paedlatrios, Dlabetology and Endoorinology

\section{Introduction:}

Alopecia areata affects $0,1-0,2 \%$ of population. It's pathogenesis has not yet been discovered, but genetic, autoimmune, vascular, psychogenic or neurological factors are being considered. It can occur in any age, but appears most commonly in late childhood, teenage or early adulthood. Alopecia areata commonly coexists with other diseases such as asthma, allergic rhinitis, atopic dermatitis, thyroid diseases and autoimmune diseases such as thyroiditis and vitiligo.

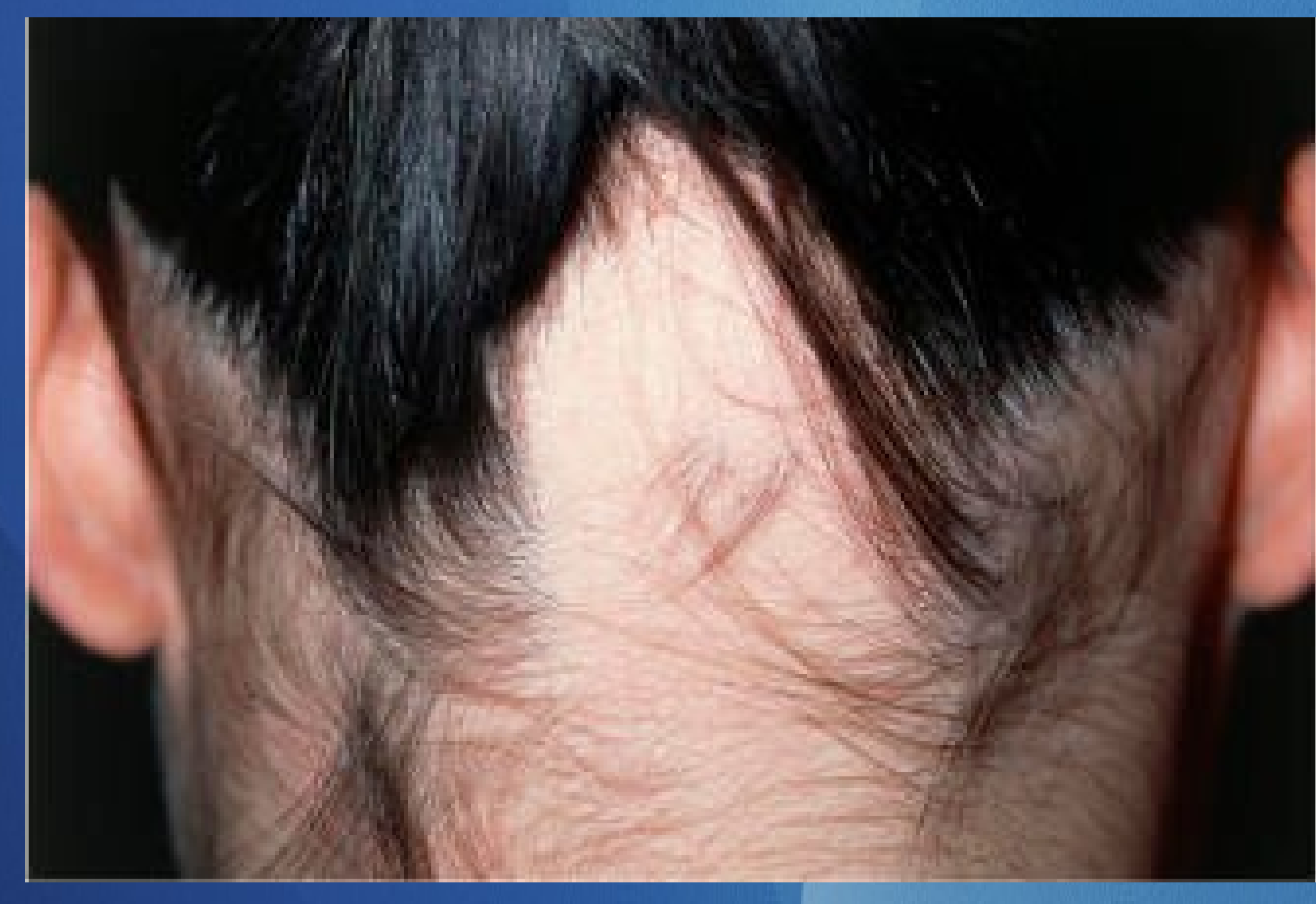

$\begin{array}{ll}\text { TGA } & >200 \mathrm{RU} / \mathrm{ml}(\mathrm{N}:<20 \mathrm{RU} / \mathrm{ml}) \\ \text { TPO-Ab } & 277,58 \mathrm{IU} / \mathrm{ml}(\mathrm{N}:<5,61 \mathrm{IU} / \mathrm{ml}) \\ \text { TG-Ab } & 95,63 \mathrm{IU} / \mathrm{ml}(\mathrm{N}:<4,11 \mathrm{IU} / \mathrm{ml}) \\ \text { ANA-HEp } & 1: 640(\mathrm{~N}:<1: 80) \\ \text { AAA } & \text { negative }\end{array}$

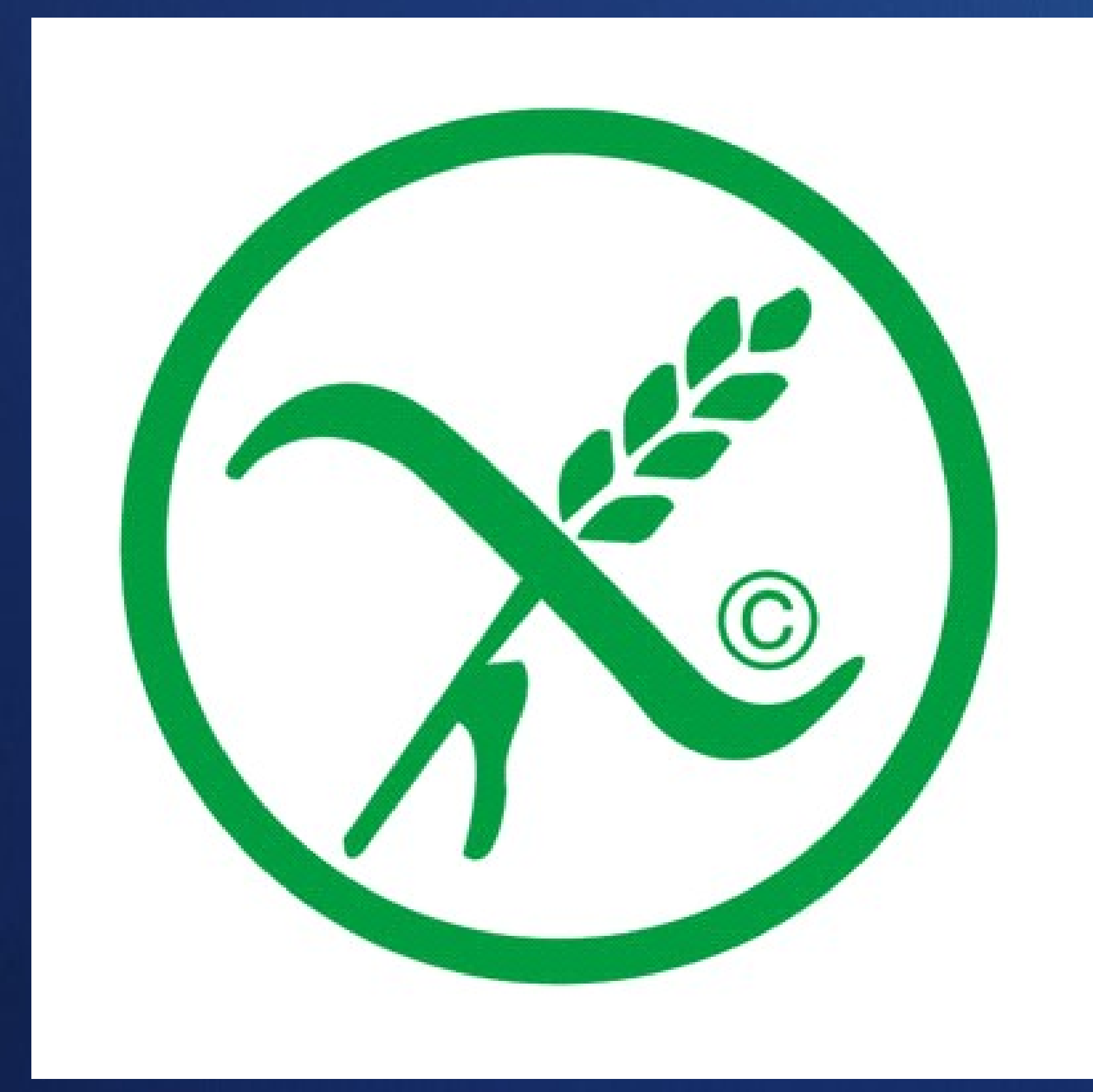

\section{Case report:}

A 14-year-old patient was admitted to hospital presenting with overweight and alopecia areata lasting for 3 years. In the past medical history at the beginnig of sympthoms also joints pain was present. Joints pain, increased CRP level and fever supported the diagnosis of reumatic fever, consequently the patient was treated with clarithromycin with a good clinical outcome, however in the last 6 months the patient has noticed elbow and shoulder joints pain again. On admission physical examination was remarkable for overweight, alopecia areata multilocularis of the scalp and enlarged thyroid gland. Laboratory findings demonstrated autoimmune thyroiditis with thyroid function tests within the normal range, increased level of antitransglutaminase antibodies as well as Hep2 antinuclear antibodies. Basing on positive HLADQ2/HLA-DQ4 coeliac disease was diagnosed. Gluten-free diet was introduced

\section{Conclusions:}

The case illustrates significance of testing patients with alopecia areata for autoimmune diseases. Alopecia areata is one of the features present in patients with autoimmune polyendocrine syndrome type 1 (APS-1). 